\title{
Multifrequency observations of the extreme X-ray weak quasar PG0043+039
}

\author{
Wolfram Kollatschny* \\ Institut für Astrophysik, Universität Göttingen, Germany \\ E-mail: wkollateastro.physik. uni-goettingen.de
}

\section{Matthias Zetzl}

Institut für Astrophysik, Universität Göttingen, Germany

E-mail: zetzleastro.physik.uni-goettingen.de

\section{Norbert Schartel}

XMM-Newton Science Operations Centre, ESA, Villafranca del Castillo, Apartado 78, 28691

Villanueva de la Cañada, Spain

E-mail: Norbert.Schartelesciops.esa.int

\begin{abstract}
PG0043+039 is an optical bright quasar $\left(\mathrm{m}_{v} \sim 15.5\right)$ at a redshift of only $\mathrm{z}=0.38$. However, it is the X-ray weakest AGN. PG0043+039 has not been detected before on deep pointed X-ray observations indicating an extreme $\alpha_{o x}$ gradient. We simultaneously took spectra of this object with the SALT and HET telescopes in the optical, and with the Hubble and XMM-Newton satellite telescopes in the UV and X-ray range in July 2013 confirming an extreme faint X-ray flux in this quasar. The optical spectrum is dominated by strong FeII blends. The UV spectrum shows besides known emission lines additional broad humps that can be explained by cyclotron lines. PG0043+039 exhibits its maximum in the overall continuum flux at around $\lambda \approx 2500 \AA$ only. Most other AGN show their maximum at higher frequencies. In combination with its intrinsic $\mathrm{X}$-ray weakness this is an indication for a not that hot inner accretion disk in PG0043+039 in comparison to most other AGN. Magnetic fields - of the order of $\times 10^{8} \mathrm{G}-$ might be responsible for the prevention of the formation of an (inner) accretion disk in PG0043+039 (see Paper I).
\end{abstract}

SALT Science Conference 2015 -SSC2015-

1-5 June, 2015

Stellenbosch Institute of Advanced Study, South Africa

* Speaker. 


\section{Introduction}

PG0043+039 is an optical luminous quasar $\left(\mathrm{M}_{B}=-26.11\right)$ at a not that distant redshift of $\mathrm{z}=0.38$. Therefore, it was surprising that this quasar has not been detected before in X-ray surveys nor in deep pointed X-ray observations. An optical spectrum of this object from the Palomar-Green sample has been published by Boroson \& Green [2]. Later on, PG0043+039 has been identified as a weak broad absorption line (BAL) quasar by Bahcall et al. [1]. Turnshek et al. [8, 9] achieved UVspectra of this object with the Hubble Space Telescope (HST) in addition to ground-based optical spectrophotometry. They found that this object reveals a number of atypical properties relative to normal non broad absorption line (BAL) quasars. Inspection of deep images taken with the XMMNewton satellite in 2005 did not reveal an X-ray counterpart for PG0043+039. Czerny et al. [3] derived only an upper limit of $<8.6 \times 10^{-16} \mathrm{ergs} \mathrm{s}^{-1} \mathrm{~cm}^{-2}$ for the source flux. We simultaneously took optical (SALT, HET), ultraviolet (HST), and X-ray spectra (XMM-Newton) in 2013 to explore the extreme X-ray weakness and furthermore to study whether this object shows other peculiarities as well. More details can be found in Kollatschny et al. [4, 5] (Paper I,II).

\section{Observations}

We took optical spectra of PG0043+039 with the 10m Southern African Large Telescope (SALT) and with the 9.2m Hobby-Eberly Telescope (HET) at McDonald Observatory nearly simultaneously to the ultraviolet (HST) and X-ray observations in July, 2013 under photometric conditions. The SALT spectrum has been taken with the Robert Stobie Spectrograph (RSS) attached to the telescope using the pg0900 grating with a 1.5 arcsec wide slit. We covered the wavelength range from 6445 to $9400 \AA$ at a spectral resolution of $4.8 \AA$ (FWHM). This wavelength region corresponds to 4653 to $6786 \AA$ in the rest frame of the galaxy. The exposure time was 2200 seconds which yielded a S/N of 118 at $7000 \AA$. The second optical spectrum was taken with the Marcario Low Resolution Spectrograph (LRS) mounted at the prime focus of the Hobby-Eberly Telescope. This spectrum covers the wavelength range from $4390 \AA$ to $7275 \AA$ (LRS grism 2 configuration) with a spectral resolution of $8.2 \AA$ (FWHM). That wavelength region corresponds to 3170 to $5250 \AA$ in the rest frame of the galaxy. The spectrum has been taken with an exposure time of 1500 seconds which yielded a S/N of 102 at $5100 \AA$.

We observed PG0043+039 over one full orbit with the Hubble Space telescope on July 18th, 2013. We used the COS/NUV spectrograph with the G140L grating and an 2.5 arcsec aperture. This spectral set covers the wavelength region from $\sim 1140 \AA$ to $\sim 2150 \AA$. This range corresponds to $\sim 800 \AA$ to $\sim 1550 \AA$ in the rest frame of the galaxy.

PG0043+039 was observed with XMM-Newton on the same date. We obtained clean exposure times of $14.5 \mathrm{ks}$ for pn, $29.0 \mathrm{ks}$ for MOS 1 and $31.3 \mathrm{ks}$ for MOS 2. We added the pn, MOS 1 and MOS 2 spectra for getting a higher S/N ratio. Finally we obtained a flux of F(2.0 $10.0 \mathrm{keV})=1.80_{-0.3}^{+0.3} \times 10^{-14} \mathrm{ergs} \mathrm{cm}^{2} \mathrm{~s}^{-1}$.

\section{Results}

A combined optical spectrum of PG0043+039 - composed of the SALT and HET spectra - is shown in Fig. 1. The blue spectrum taken with the HET telescope and the red spectrum taken with the SALT telescope perfectly overlap in the common $\mathrm{H} \beta$ region. Furthermore, both 


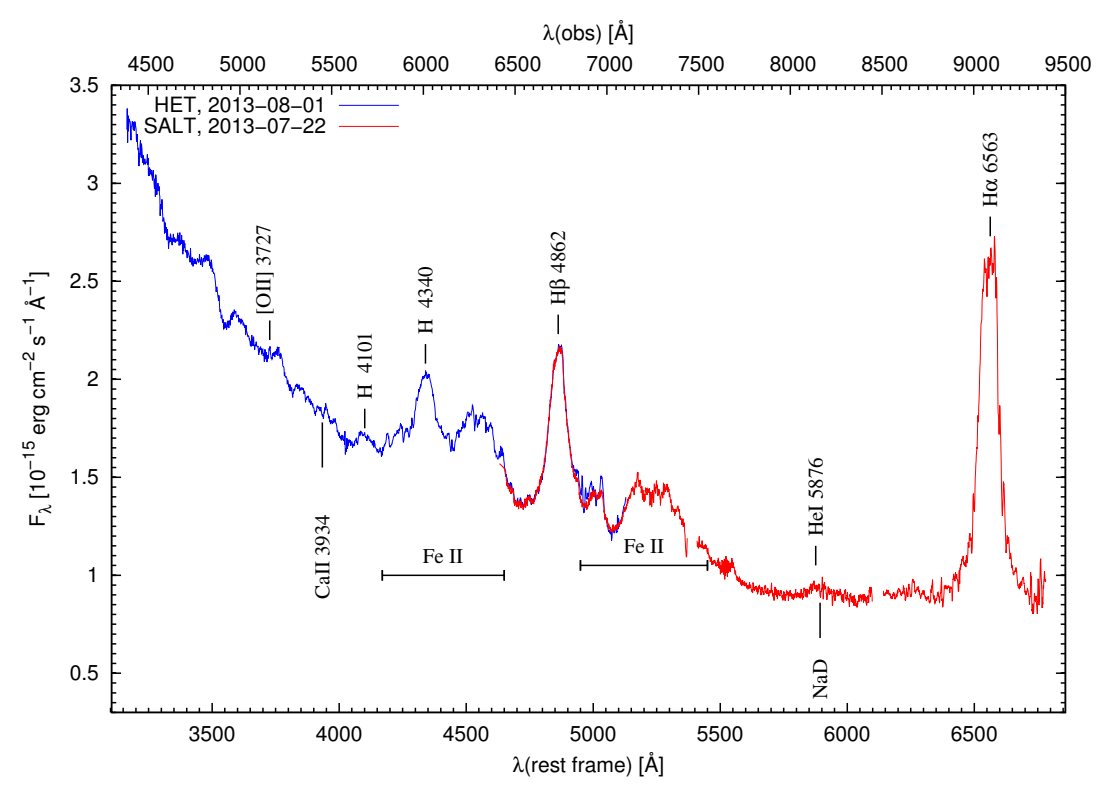

Figure 1: Combined optical spectra of PG0043+039 taken with the SALT and HET telescopes.

spectra have a similar $\mathrm{S} / \mathrm{N}$ ratio. The observed total spectral range in the optical corresponds to a rest frame spectrum from $\sim 3170 \AA$ to $\sim 6790 \AA$. The Balmer lines dominate the optical spectrum. In addition, PG0043+039 is a strong optical FeII emitter. This has been noted before by Turnshek et al. [8]. There are no indications for the presence of the narrow [O III] $\lambda 5007, \lambda 4959$ lines. But we clearly detect a weak narrow $[\mathrm{O}$ II $] \lambda 3727$ line. The underlying continuum of the optical spectrum shows a strong blue gradient corresponding to a power-law continuum $\mathrm{F}_{v} \sim v^{\alpha}$ with $\alpha=-2.55 \pm 0.02$ (see Fig.3).

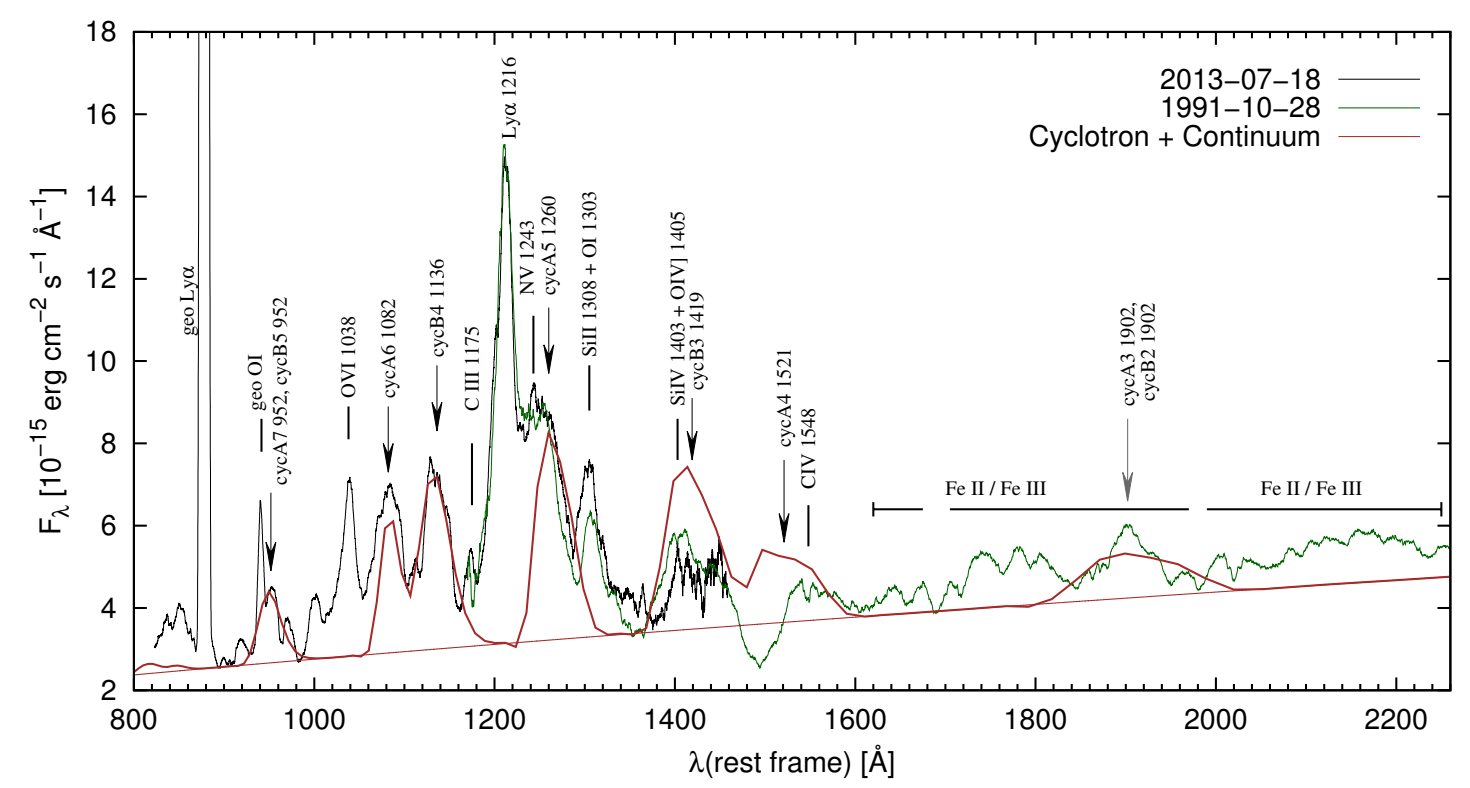

Figure 2: Combined UV spectra of PG0043+039 taken with the HST in the years 2013 and 1991.

The UV spectrum of PG0043+039 - taken with the HST-FOS in July 2013 - is shown in 
Fig. 2. Overlaid is a second UV spectrum of PG0043+039 that has been taken before with the HST in 1991 (Turnshek et al. [8]). This spectrum covered the intrinsic wavelength range from $\sim 1130 \AA$ to $\sim 2300 \AA$. The continuum and line fluxes increased by a factor of $\sim 1.8$ between the years 1991 and 2013 when comparing the overlapping wavelength range of both UV spectra (Kollatschny et al. [5]). We multiplied the UV spectrum taken in 1991 with this factor 1.8 to match the UV observations of 2013. Indicated are the identifications of the strongest UV emission lines (e.g. O vi $\lambda 1038$, Ly $\alpha, N \vee \lambda 1243$ ), of two geo-coronal lines, as well as of the cyclotron systems A and B with their 2nd to 7th harmonics. For more details see Kollatschny et al. [4]. Overlaid is a power-law continuum $\mathrm{F}_{\lambda} \sim \lambda^{\alpha}$ with $\alpha=0.67 \pm 0.02$ for fitting the $\mathrm{UV}$ continuum.

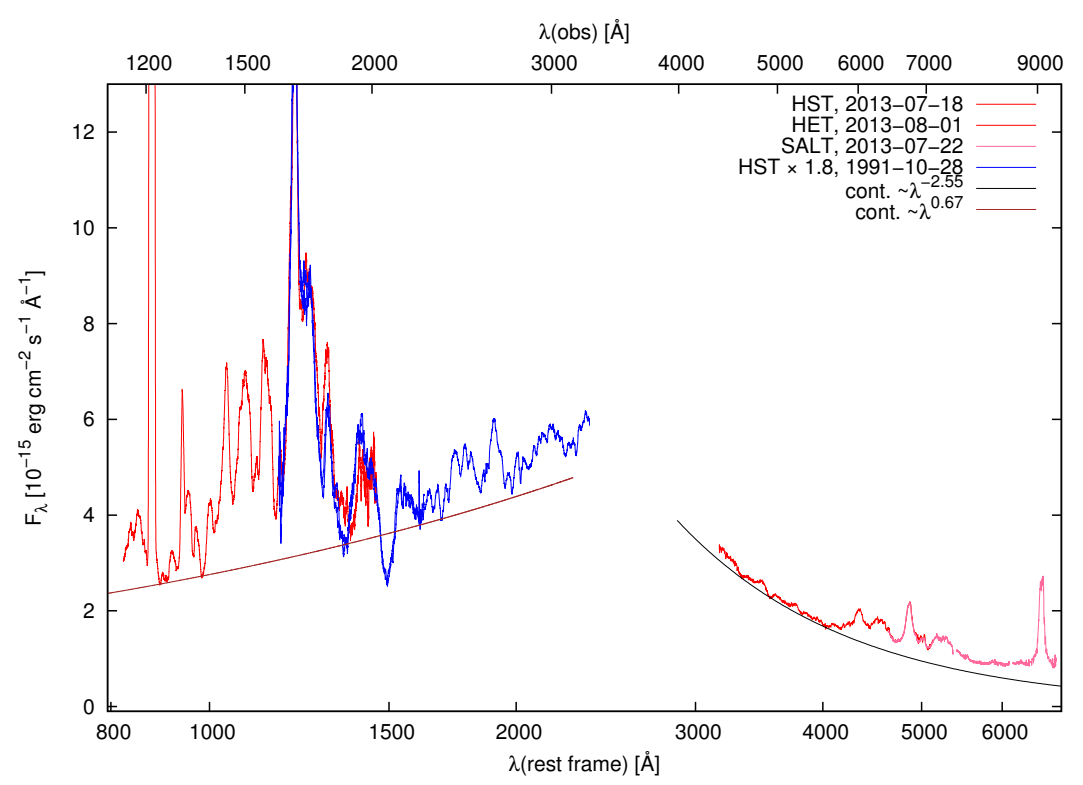

Figure 3: Combined optical and ultraviolet spectra of PG0043+039 taken in the years 2013 (red lines) and 1991 (blue line). Power-law continua are shown separately for the optical and ultraviolet spectral ranges in addition.

We present in Fig. 3 a combination of our optical and UV spectra of PG0043+039. Power-law continua adapted to the UV as well as optical spectral ranges are displayed in addition. There is a clear maximum in the overall continuum flux at around $2500 \AA$ (rest frame): The flux is getting weaker towards both shorter and longer wavelengths. We compute a very steep UV power-law slope parameter $\alpha_{o x}=-2.55 \pm 0.3$ based on the near as well as far UV data. A maximum in the transition region between the optical and UV spectral range has been mentioned before by Turnshek et al. [8].

Fig. 4 presents the observed optical-UV-X-ray spectral distribution of PG0043+039 in July, 2013. Given are the optical spectra (taken with the SALT and HET telescopes), the UV and far-UV spectra taken with the HST, and the X-ray flux taken with the XMM-Newton satellite. The extrapolation of the UV power-law continuum $\mathrm{F}_{v} \sim v^{\alpha}$ with $\alpha=-2.67 \pm 0.02$. perfectly corresponds to the extreme faint X-ray flux. The dashed line shows the mean composite SED derived from a sample of luminous quasars (Richards et al. [6]) for comparison. 


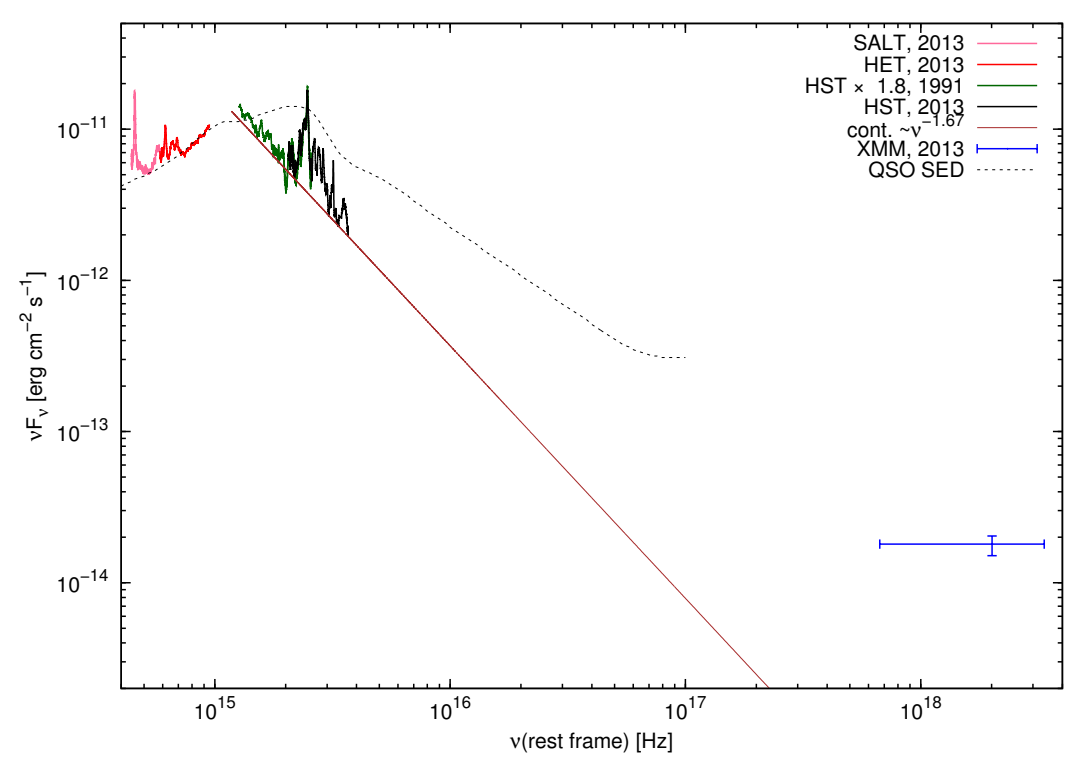

Figure 4: Multiwavelength (opt-UV-X-ray) spectral distribution of PG0043+039 with an extrapolation of the UV power-law continuum. The mean spectral distribution of a luminous quasar sample is given by the dashed line.

\section{Discussion}

We presented spectral observations of PG0043+039 taken simultaneously in the optical, in the $\mathrm{UV}$, and in the X-ray spectral range. The spectra obtained in the individual frequency ranges are peculiar. This also applies to the overall spectral distribution. The optical spectrum is dominated by strong FeII bands - similar to the peculiar AGN Mrk231. However, there are clear differences between these objects in the UV spectral range. Mrk231 (e.g. Veilleux et al. [7]) does not show the strong UV emission bumps seen in PG0043+039 (Paper I). We attributed these broad humps to cyclotron emission. The modeling of these humps with cyclotron lines could explain their wavelength positions, their relative distances as well as their relative intensities.

Based on our simultaneous observations in different frequency ranges we could show that the X-ray faintness of PG0043+039 is not simulated by variations but is an intrinsic property. The extreme UV/X-ray weakness of PG0043+039 is shown in Fig. 4 in comparison to the mean spectral distribution of luminous QSOs (Richards et al. [6]). PG0043+039 exhibits its maximum flux in the overall continuum distributiom at $\lambda \approx 2500 \AA$ only. Most other AGN show their maximum at higher frequencies (see Fig.4). In combination with its intrinsic X-ray weakness this is an indication for a not that hot inner accretion disk in PG0043+039 compared to most other AGN. Magnetic fields - of the order of $\times 10^{8} \mathrm{G}$ - might be responsible for the prevention of the formation of an (inner) accretion disk in PG0043+039 (see Paper I).

\section{Acknowledgments}

We thank M. Santos-Lleó for her contribution to this common work. This work has been supported by the DFG grant Ko 857/32-2. 


\section{References}

[1] Bahcall, J. N., et al. 1993, ApJS, 87, 1

[2] Boroson, T. A., Green, R. F. 1992, ApJS, 80, 109

[3] Czerny, B., Siemiginowska, A., Janiuk, A. et al.. 2008, MNRAS, 386, 1557

[4] Kollatschny, W., Schartel, N., Zetzl, M., et al. 2015, A\&A, 577, L1 (Paper I)

[5] Kollatschny, W., Schartel, N., Zetzl, M., et al. 2016, A\&A, 585, 18 (Paper II)

[6] Richards, G. T., et al., 2006, ApJS, 166, 470

[7] Veilleux, S., Trippe, M., Hamann, F., et al. 2013, ApJ, 764, 15

[8] Turnshek, D. A., Espey, B. R., Kopko, JR., M., et al. 1994, ApJ, 428, 93

[9] Turnshek, D. A., Monier, E. M., Sirola, C. J., et al. 1997, ApJ, 476, 40 